\title{
Producing Type lax supernovae from a specific class of helium-ignited WD explosions
}

\author{
B. Wang ${ }^{1,2}$, S. Justham ${ }^{3}$, and Z. Han $^{1,2}$ \\ 1 Yunnan Observatories, Chinese Academy of Sciences, 650011 Kunming, PR China \\ e-mail: [wangbo; zhanwenhan]@ynao.ac.cn \\ 2 Key Laboratory for the Structure and Evolution of Celestial Objects, Chinese Academy of Sciences, 650011 Kunming, PR China \\ ${ }^{3}$ National Astronomical Observatories, Chinese Academy of Sciences, 100012 Beijing, PR China \\ e-mail: sjustham@bao.ac.cn
}

Received 17 July 2013 / Accepted 8 October 2013

\section{ABSTRACT}

\begin{abstract}
Context. It has recently been proposed that one subclass of Type Ia supernovae (SNe Ia) is sufficiently distinct and sufficiently common to be classified separately from the bulk of SNe Ia; the suggested class name is Type Iax supernovae (SNe Iax), after SN 2002cx. However, their progenitors are still uncertain.

Aims. We study whether the population properties of this class might be understood if the events originate from a subset of subChandrasekhar mass explosions. In this potential progenitor population, a carbon-oxygen white dwarf (CO WD) accumulates a helium layer from a non-degenerate helium star; ignition of that helium layer then leads to ignition of the CO WD.

Methods. We incorporated detailed binary evolution calculations for the progenitor systems into a binary population synthesis model to obtain rates and delay times for these events.

Results. The predicted Galactic event rate of these explosions is $\sim 1.5 \times 10^{-3} \mathrm{yr}^{-1}$ according to our standard model, in good agreement with the measured rates of SNe Iax. In addition, predicted delay times are $\sim 70 \mathrm{Myr}-800 \mathrm{Myr}$, consistent with the fact that most of SNe Iax have been discovered in late-type galaxies. If the explosions are assumed to be double detonations - following current model expectations - then, based on the CO WD masses at explosion, we also estimate the distribution of resulting SN brightness $\left(-13 \gtrsim M_{\mathrm{bol}} \gtrsim-19 \mathrm{mag}\right)$, which can reproduce the empirical diversity of SNe Iax.

Conclusions. We speculate on why binaries with non-degenerate donor stars might lead to SNe Iax if similar systems with degenerate donors do not. We suggest that the high mass of the helium layer necessary for ignition at the lower accretion rates typically delivered from non-degenerate donors might be necessary to produce SN 2002cx-like characteristics, perhaps even by changing the nature of the CO ignition.
\end{abstract}

Key words. binaries: close - stars: evolution - supernovae: general

\section{Introduction}

Type Ia supernovae (SNe Ia) play an important role in astrophysics, especially in the studies of cosmic evolution and galactic chemical evolution. It has been proposed that Type Iax supernovae ( $\mathrm{SNe}$ Iax) form a distinct subclass of sub-luminous $\mathrm{SNe}$ Ia, containing $\mathrm{SNe}$ that resemble the prototype event SN 2002cx (Li et al. 2003; Foley et al. 2013). These SNe Iax are spectroscopically similar to SNe Ia, but have lower maximumlight velocities $\left(2000 \lesssim|v| \lesssim 8000 \mathrm{~km} \mathrm{~s}^{-1}\right)$, typically lower peak magnitudes $\left(-14.2 \gtrsim M_{\mathrm{V}} \gtrsim-18.9 \mathrm{mag}\right)^{1}$, and maximumlight spectra that typically resemble those of the bright 1991Tlike events. Since the estimated rate of SNe Iax is roughly one third of the SN Ia rate, they are relatively common astrophysical events, although only 25 members of the class have currently been identified (Foley et al. 2013).

Obviously, SNe Iax appear not to obey the standard luminosity-width relation of SNe Ia (Foley et al. 2013, and references therein); this would affect any use of them as distance indicators, although the fact that SNe Iax are low-luminosity events means that this is unlikely to be problematic in practice. However, perhaps more importantly, this behaviour of SNe Iax also provides an opportunity to help us understand the

\footnotetext{
A typical peak brightness of normal SNe Ia is about -19 mag, and with a spread in brightness of $\sim 1$ mag (e.g., Benetti et al. 2005).
}

physics of thermonuclear supernovae in general, since whatever mechanism produces SNe Iax leads to an alternative family of lightcurve shapes from standard SNe Ia. Deducing the progenitors of these explosions should help us to understand how this family of explosions differs from standard SNe Ia. Observations support the supposition that $\mathrm{SNe}$ Iax are from thermonuclear explosions of carbon-oxygen white dwarfs (CO WDs), due to the evidence of $\mathrm{C} / \mathrm{O}$ burning in their maximum-light spectra (Foley et al. 2013). Generally, SNe Ia lack helium in their spectra, yet two SNe Iax show strong helium lines in their spectra, and so there might be helium in their progenitor systems. However, there is no hydrogen in any SN Iax spectra, and significantly less hydrogen than helium is typically required to cause a signature in an SN spectra (e.g., Hachinger et al. 2012). That spectral evidence might suggest that in the SN Iax progenitor systems a CO WD is accreting from a non-degenerate helium star or a He WD. Of those, the CO WD + He WD systems would exist in old stellar populations as well as in young populations, which is inconsistent with the observation that most of SNe Iax have been discovered in late-type galaxies (e.g., Valenti et al. 2009; Foley et al. 2013) $)^{2}$. However, CO WD + He star systems

2 One of the SNe Iax (i.e., SN 2008ge) was discovered in an old environment, and the environment of the rest is very young, comparable with that of Type IIp core-collapse SNe (see Lyman et al. 2013). 
are expected primarily in young stellar populations, as has been observed. In this article, we will investigate the population properties of CO WD + He star systems and ask whether they are consistent with being the progenitors of SNe Iax.

A CO WD can accrete material from a helium star to increase its mass until it ignites near to the Chandrasekhar mass limit (e.g., Wang et al. 2009a). However, standard Chandrasekhar mass explosion models have difficulty in reproducing the low luminosities of SNe Iax (Hillebrandt \& Niemeyer 2000). It is therefore natural to consider whether SNe Iax might be produced by sub-Chandrasekhar mass explosions, in which the explosion of a CO WD is triggered by the detonation of a substantial surface layer of accreted helium (e.g., Nomoto 1982; Woosley et al. 1986). However, the details of what happens following burning of the helium layer are still unclear. If helium ignites at the bottom of the helium layer, this may result in an event known as helium double-detonation, during which one detonation wave propagates outward through the helium layer, whereas an inward propagating pressure wave compresses the $\mathrm{CO}$ core and leads to ignition followed by an outward detonation (e.g., Livne 1990; Woosley \& Weaver 1994). Helium double-detonation subChandrasekhar mass explosions have previously been considered as promising explanations for standard SNe Ia (e.g., Branch et al. 1995; Höflich \& Khokhlov 1996), but modern models disagree over which known $\mathrm{SNe}$, if any, these systems might produce (e.g., Fink et al. 2007, 2010; Kromer et al. 2010; Woosley \& Kasen 2011; Sim et al. 2012). In principle, there is no reason why some double-detonation events could not produce normal $\mathrm{SNe}$ Ia whilst other similar progenitor systems lead to SNe Iax.

Generic double-detonation scenarios have previously been suggested for SNe Iax, but inconclusively (e.g., Foley et al. 2013, and references therein). We note that Foley et al. (2009) suggested that an alternative explosion model might be responsible for SNe Iax, specifically a failed deflagration model; this model has successfully explain the observed properties of SNe Iax in some aspects, and could even explain the low ejecta-mass (e.g., Foley et al. 2013). In models like this, the accreting WD would survive and potentially possess peculiar observational properties (see also Jordan et al. 2012; Kromer et al. 2013). Perhaps the main reason to doubt double-detonation scenarios for SNe Iax is that double-detonation models have tended not to produce the low-velocity ejecta characteristic of SNe Iax. However, recent work has generally suggested that the diversity of observables which result from this class of models is rather sensitive to details of the pre-ignition conditions (see especially Kromer et al. 2010; Woosley \& Kasen 2011; Sim et al. 2012); we therefore see no fundamental reason why the peculiar properties of SNe Iax might not be matched by future developments of such calculations. In particular, we will speculate that the relatively massive layer of accreted helium that is expected to be present at explosion for these progenitor systems might lead to the observed properties of SNe Iax. Determining the nature of SN Iax progenitors should certainly help to refine explosion models.

A second argument against double detonations as an explanation for SNe Iax is provided by SN 2008ha. This extreme member of the SN Iax class was inferred to have a very low ejecta mass $\left(\sim 0.3 M_{\odot}\right.$; Foley et al. 2010). If that ejecta mass is correct, then this event might be explained by a He-shell explosion (e.g., Bildsten et al. 2007; Shen \& Bildsten 2009; Waldman et al. 2011), but a complete double detonation seems very unlikely. In that case, then SN 2008ha would need to be explained as a different type of event. However, that would not necessarily mean that this particular progenitor population could not have produced SN 2008ha, if ignition of the helium shell in these systems can lead to less complete burning of the CO WD, perhaps even including failed deflagrations. Whilst recent theoretical work broadly finds that double detonations can be robustly triggered, several subtleties still remain to be solved (Moll \& Woosley 2013; Shen \& Bildsten 2013). For convenience, most of this work is written as if ignition of the helium layer in this particular progenitor population inevitably leads to a double detonation, but the reality may well be more complex.

There are numerous complications, including that the properties of the helium layer that lead to helium ignition itself are expected to be a function of CO WD mass and accretion rate (e.g., Bildsten et al. 2007; Shen \& Bildsten 2009). Heating from differential rotation in the accreted layer provides further uncertainty (Yoon \& Langer 2004). Transients have also been observed that are consistent with the detonation of a thick helium layer on a WD which does not lead to a double detonation (Poznanski et al. 2010; Kasliwal et al. 2010; Perets et al. 2011). Previous studies have often made the simplification that, for sufficiently massive CO WDs, a helium layer with mass $0.1 M_{\odot}$ can ignite and lead to a double detonation (e.g., Ivanova \& Taam 2004; Ruiter et al. 2011), and Fink et al. (2007, 2010) support the position that layers of that mass can produce a double-detonation.

We note that $\mathrm{SNe}$ Iax may be a bridge between normal SNe Ia and the SN 2005E-like objects, for which helium-rich thermonuclear explosions have also been proposed as an explanation (e.g., Perets et al. 2010); SN 2005E-like objects share many characteristics with SNe Iax, including luminosity, velocity, and ejecta-mass, etc (e.g., Sullivan et al. 2011; Kasliwal et al. 2012). However, these SNe appear to come from old stellar populations, in contrast to SNe Iax. Even more enigmatically, the known examples from SN 2005E-like objects suggest that they are preferentially produced in the outer regions of galaxies (e.g., Yuan et al. 2013; Lyman et al. 2013).

Overall, determining the progenitors of SNe Iax could distinguish between theoretical models which are largely separated by precise details of thermonuclear explosion physics at WD densities.

The paper is organized as follows. In Sect. 2, we further describe our assumptions and binary evolution calculations. The binary evolutionary results are shown in Sect. 3. We describe the binary population synthesis (BPS) method in Sect. 4, and present the BPS results in Sect. 5. Finally, a discussion and summary are given in Sect. 6.

\section{Binary evolution calculations}

Employing Eggleton's stellar evolution code (Eggleton 1971, 1972, 1973; later updated by Han et al. 1994; Pols et al. 1998), we calculated the evolution of the CO WD + He star systems. Roche lobe overflow (RLOF) is treated within the code as described by Han et al. (2000). We set the ratio of mixing length to local pressure scale height, $\alpha=l / H_{\mathrm{p}}$, to be 2.0. In our calculations, the initial helium star models are composed of helium abundance $Y=0.98$ and metallicity $Z=0.02$. Orbital angular momentum loss due to gravitational wave radiation (GWR) is included by adopting a standard formula presented by Landau \& Lifshitz (1971),

$\frac{\mathrm{d} \ln J_{\mathrm{GR}}}{\mathrm{d} t}=-\frac{32 G^{3}}{5 c^{5}} \frac{M_{\mathrm{WD}} M_{2}\left(M_{\mathrm{WD}}+M_{2}\right)}{a^{4}}$,

where $G, c, M_{\mathrm{WD}}$, and $M_{2}$ are the gravitational constant, vacuum speed of light, the mass of the accreting WD, and the mass of the companion He star, respectively. 

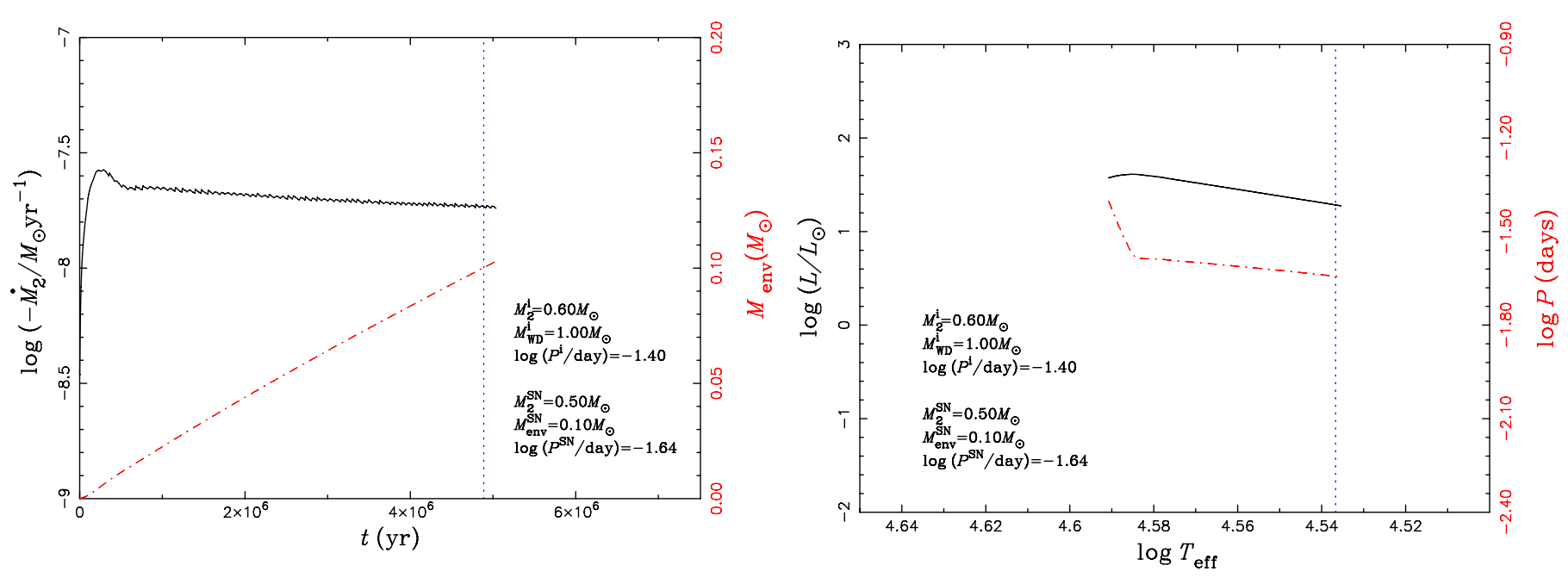

Fig. 1. A representative example of binary evolution calculations. Left panel: the solid and dash-dotted curves show the mass-transfer rate and the mass of the helium layer on the WD varying with time after the helium star fills its Roche lobe, respectively. Right panel: the evolutionary track of the donor star is shown as a solid curve and the evolution of orbital period is shown as a dash-dotted curve. Dotted vertical lines in both panels indicate the position where the double detonation may happen. The initial binary parameters and the parameters at the moment of the SN explosion are given in these two panels.

In the double-detonation model, the helium star transfers some of its material onto the surface of the WD, which increases the mass of the WD as a consequence. If the masstransfer rate onto the WD from the helium star is higher than $4 \times 10^{-8} M_{\odot} \mathrm{yr}^{-1}$, stable burning allows the CO mass of the WD to increase (Woosley et al. 1986; see also Wang et al. 2009a, and references therein). For lower accretion rates $\left(1 \times 10^{-9} M_{\odot} \mathrm{yr}^{-1} \lesssim\right.$ $\left|\dot{M}_{2}\right| \lesssim 4 \times 10^{-8} M_{\odot} \mathrm{yr}^{-1}$ ), a thick layer of helium is believed to grow on the surface of the WD. When the mass-transfer rate drops even lower $\left(\left|\dot{M}_{2}\right|<1 \times 10^{-9} M_{\odot} \mathrm{yr}^{-1}\right)$, the flash when the helium layer ignites has been suggested to be too weak to initiate a carbon detonation, which results in only a single helium detonation wave propagating outward (e.g., Nomoto et al. 1982) ${ }^{3}$. For accretion rates of a few times $10^{-8} M_{\odot} \mathrm{yr}^{-1}$, Yoon \& Langer (2004) have argued that heating by frictional dissipation significantly reduces the possibility of a helium detonation, leading to some uncertainty in these mass-transfer rate boundaries.

According to recent hydrodynamic simulations, the minimum WD mass for carbon burning might be $\sim 0.8 M_{\odot}$, since the detonation of the CO WD may be not triggered for lower mass (e.g., Sim et al. 2012). We also expect that the initial CO WD masses are below $\approx 1.1 M_{\odot}$ as more massive WDs at formation - usually consist of oxygen and neon (i.e., ONe WDs). In principle, the WD could increase its CO mass by accreting helium as long as the mass-transfer rate is higher than $4 \times 10^{-8} M_{\odot} \mathrm{yr}^{-1}$, but this rarely occurs in our calculations; only helium donors with masses above $\approx 1.1 M_{\odot}$ lead to an increase in $\mathrm{CO}$ mass. Following the previous work described in the introduction, we assume that a double detonation occurs when a helium layer with mass $0.1 M_{\odot}$ accumulates on the surface of the WD. We note that the double-detonation model seems difficult to reconcile with the low ejecta mass reported for SN 2008ha (for a detailed discussion see the introduction).

We incorporated the prescriptions above into Eggleton's stellar evolution code and followed the evolution of an ensemble of $\mathrm{CO}$ WD + He star systems. The mass lost from these systems

\footnotetext{
3 Our upper accretion rate limit only directly affects a small fraction of our population. The lower limit is uncertain, but might approximately be justified in an additional way, i.e., that below these rates the helium layer mass needed for ignition is normally too large to be reached (e.g., Shen \& Bildsten 2009).
}

is assumed to take away the specific orbital angular momentum of the accreting WD. We have calculated the evolution of about $600 \mathrm{WD}+\mathrm{He}$ star systems, thereby obtaining a large, dense model grid. The initial mass of the helium donor stars, $M_{2}^{\mathrm{i}}$, ranges from $0.3 M_{\odot}$ to $1.3 M_{\odot}$; the initial mass of the CO WDs, $M_{\mathrm{WD}}^{\mathrm{i}}$, is from $0.8 M_{\odot}$ to $1.10 M_{\odot}$; the initial orbital period of the binary systems, $P^{\mathrm{i}}$, changes from the minimum value, at which a helium zero-age main-sequence star would fill its Roche lobe to $\sim 0.2 \mathrm{~d}$, where the helium star fills its Roche lobe at the end of the helium MS.

\section{Binary evolution results}

\subsection{An example of binary evolution calculations}

In Fig. 1, we present a representative example of binary evolution calculations for the double-detonation model. The left panel shows the mass-transfer rate and the mass of the WD envelope varying with time after the helium star fills its Roche lobe, whereas the right panel is the evolutionary track of the helium donor star in the Hertzsprung-Russell diagram, where the evolution of the orbital period is also shown. The WD + He star binary starts with $\left(M_{2}^{\mathrm{i}}, M_{\mathrm{wD}}^{\mathrm{i}}, \log \left(P^{\mathrm{i}} /\right.\right.$ day $\left.)\right)=(0.60,1.0,-1.4)$, where $M_{2}^{\mathrm{i}}$ and $M_{\mathrm{WD}}^{\mathrm{i}}$ are the initial masses of the helium star and of the CO WD in solar mass, and the $P^{\mathrm{i}}$ is the initial orbital period in days.

Because of the short initial orbital period $(0.04 \mathrm{~d})$, angular momentum loss induced by GWR is large, which leads to the rapid shrinking of the orbital separation. After about 11 million years, the helium star begins to fill its Roche lobe whilst it is still in the helium core-burning stage. The mass-transfer rate is stable and with a low rate of $\sim 2 \times 10^{-8} M_{\odot} \mathrm{yr}^{-1}$, which results in the formation of a helium layer on the surface of the CO WD. After about 5 million years, the mass of the helium layer increases to $0.1 M_{\odot}$, at which point a double detonation is assumed to occur. At this moment, the mass of the helium star is $M_{2}^{\mathrm{SN}}=0.50 M_{\odot}$ and the orbital period is $\log \left(P^{\mathrm{SN}} /\right.$ day $)=-1.64$.

\subsection{Initial parameters for $\mathrm{SN}$ la progenitors}

Figure 2 shows the initial contours for producing $\mathrm{SNe}$ Ia in the $\log P^{\mathrm{i}}-M_{2}^{\mathrm{i}}$ plane for various $\mathrm{WD}$ masses, i.e., $M_{\mathrm{WD}}^{\mathrm{i}}=0.8$, 


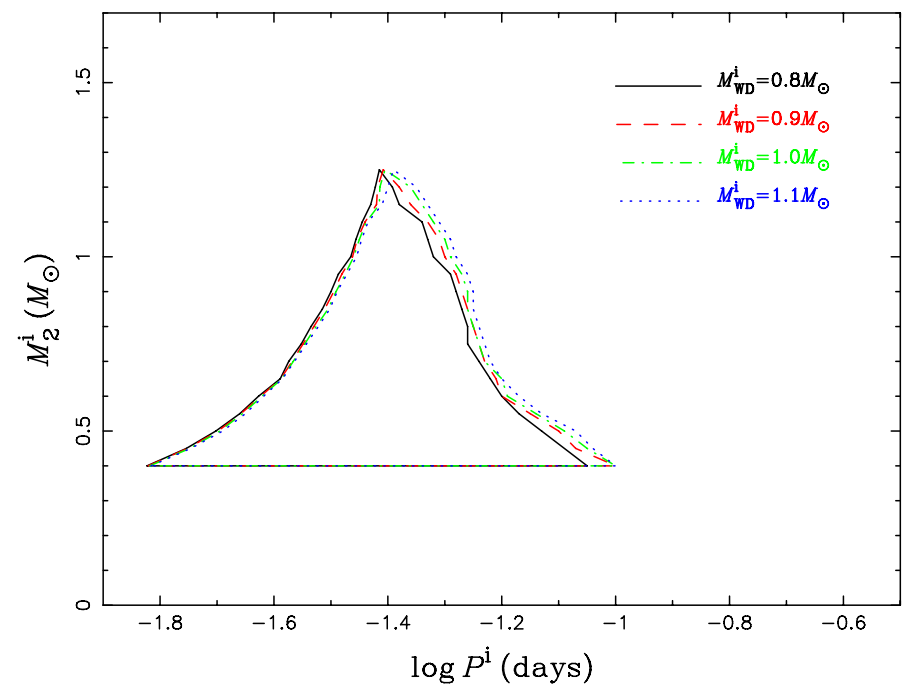

Fig. 2. Regions in the initial orbital period-secondary mass plane $\left(\log P^{\mathrm{i}}, M_{2}^{\mathrm{i}}\right)$ for $\mathrm{WD}+\mathrm{He}$ star binaries that produce $\mathrm{SNe}$ Ia for various initial WD masses.

$0.9,1.0$, and $1.1 M_{\odot}$, where $P^{\mathrm{i}}$ and $M_{2}^{\mathrm{i}}$ are the initial orbital period and the initial mass of the helium donor star, respectively. From this figure we can see that the contours are slightly shifted to higher periods with the increase in initial WD masses. This is because a helium star with a specific mass has a smaller Roche-lobe radius with a more massive WD companion. The left boundaries of the contours are set by the condition that RLOF starts when the secondary is on the helium zero-age mainsequence, whereas systems beyond the right boundary will experience a high mass-transfer rate when the helium star evolves to the subgiant stage that is not suitable for a double-detonation explosion. The upper boundaries are set mainly by a high masstransfer rate due to orbit decay induced by GWR and large massratio, which makes the WD grow in mass to the Chandrasekhar mass limit (see Wang et al. 2009a). The lower boundaries are where the mass-transfer rate $\dot{M}_{2}$ is higher than $1 \times 10^{-9} M_{\odot} \mathrm{yr}^{-1}$ for just long enough to produce a $0.1 M_{\odot}$ helium layer on the surface of the CO WD.

\section{Binary population synthesis}

In this double-detonation model, the progenitor systems containing a CO WD + He star in a close binary have most likely emerged from the common-envelope (CE) evolution of a giant binary system. We use the standard energy equations to calculate the output of the CE phase (following Webbink 1984), in which there are two uncertain parameters, $\alpha_{\text {ce }}$ (the CE energetic ejection efficiency) and $\lambda$ (a structure parameter that depends on the evolutionary stage of the donor star and the definition of the core-envelope boundary). As in previous studies (e.g., Wang et al. 2010a; Wang \& Han 2010a,b), we combine $\alpha_{\text {ce }}$ and $\lambda$ into a single free parameter $\alpha_{\mathrm{ce}} \lambda$, and show results for two values, 0.5 and 1.5 .

To obtain event rates and delay times for the doubledetonation model, we performed a series of Monte Carlo binary population synthesis (BPS) simulations. For each BPS realization, we used Hurley's rapid binary evolution code (Hurley et al. $2000,2002)$ to follow the evolution of $10^{7}$ sample binaries with metallicity $Z=0.02$ from star formation to the formation of the CO WD + He star systems based on three evolutionary scenarios (i.e., the He star, EAGB and TPAGB channels; for details see Wang et al. 2009b). We then apply the calculations described in
Sect. 2 and assume that if the initial parameters of a CO WD + He star system are located inside the relevant contour of Fig. 2, a double-detonation explosion occurs.

The Monte Carlo BPS simulations require as input the initial mass function (IMF) of the primary, the mass-ratio distribution, the distribution of initial orbital separations, the eccentricity distribution of binary orbit, and the star formation rate (SFR).

(1) The IMF of Miller \& Scalo (1979, MS79) is adopted. The primordial primary is generated according to the formula of Eggleton et al. (1989),

$M_{1}^{\mathrm{p}}=\frac{0.19 X}{(1-X)^{0.75}+0.032(1-X)^{0.25}}$,

where $X$ is a random number uniformly distributed in the range $[0,1]$ and $M_{1}^{\mathrm{p}}$ is the mass of the primordial primary, which ranges from $0.1 M_{\odot}$ to $100 M_{\odot}$. The studies of the IMF by Kroupa et al. (1993) and Zoccali et al. (2000) support this IMF. Alternatively, we also consider the IMF of Scalo (1986, S86),

$M_{1}^{\mathrm{p}}=0.3\left(\frac{X}{1-X}\right)^{0.55}$,

where the meanings of $X$ and $M_{1}^{\mathrm{p}}$ are similar to that of Eq. (2).

(2) The initial mass-ratio distribution of the binaries, $q^{\prime}$, is quite uncertain for binary evolution. For simplicity, we take a constant mass-ratio distribution (Mazeh et al. 1992; Goldberg \& Mazeh 1994),

$n\left(q^{\prime}\right)=1, \quad 0<q^{\prime} \leq 1$,

where $q^{\prime}=M_{2}^{\mathrm{p}} / M_{1}^{\mathrm{p}}$. This constant mass-ratio distribution is supported by the study of Shatsky \& Tokovinin (2002). Alternatively, we also consider a rising mass ratio distribution

$n\left(q^{\prime}\right)=2 q^{\prime}, \quad 0 \leq q^{\prime} \leq 1$,

and the case in which both binary components are chosen randomly and independently from the same IMF (uncorrelated).

(3) We assume that all stars are members of binaries and that the distribution of separations is constant in $\log a$ for wide binaries, where $a$ is separation and falls off smoothly at small separation,

$a \cdot n(a)=\left\{\begin{array}{lc}\alpha_{\mathrm{sep}}\left(a / a_{0}\right)^{\mathrm{m}}, & a \leq a_{0}, \\ \alpha_{\mathrm{sep}}, & a_{0}<a<a_{1},\end{array}\right.$

where $\alpha_{\text {sep }} \approx 0.07, a_{0}=10 R_{\odot}, a_{1}=5.75 \times 10^{6} R_{\odot}=0.13 \mathrm{pc}$, and $m \approx 1.2$. This distribution implies that the numbers of wide binary systems per logarithmic interval are equal, and that about 50 percent of stellar systems have orbital periods less than $100 \mathrm{yr}$ (e.g., Han et al. 1995). We note that recent studies indicate that the initial separation distribution above is reasonable for high-mass stars (e.g., Sana et al. 2012), but is not good for low-mass stars (Rahgavan et al. 2010). The SN Ia progenitor systems in this article were surely not initially low-mass systems. Moreover, since more of the progenitor primaries are closer to high-mass than low-mass, then it seems more logical to adopt the distribution for highmass stars. 
Table 1. Galactic SN Ia rates for different simulation sets in which set 2 is our standard model.

\begin{tabular}{cccccc}
\hline \hline Set & $\alpha_{\text {ce }} \lambda$ & IMF & $n\left(q^{\prime}\right)$ & ecc & $v\left(10^{-3} \mathrm{yr}^{-1}\right)$ \\
\hline 1 & 0.5 & MS79 & Constant & Circular & 0.399 \\
2 & 1.5 & MS79 & Constant & Circular & 1.542 \\
3 & 1.5 & MS79 & Constant & Uniform & 1.547 \\
4 & 1.5 & S86 & Constant & Circular & 1.007 \\
5 & 1.5 & MS79 & Rising & Circular & 1.695 \\
6 & 1.5 & MS79 & Uncorrelated & Circular & 0.158 \\
\hline
\end{tabular}

Notes. $\alpha_{\mathrm{ce}} \lambda=\mathrm{CE}$ ejection parameter; IMF = initial mass function; $n\left(q^{\prime}\right)=$ initial mass ratio distribution; ecc $=$ eccentricity distribution of binary orbit; $v=$ Galactic SN Ia rates.

(4) A circular orbit is assumed for all binaries. The orbits of semi-detached binaries are generally circularized by the tidal force on a timescale which is much smaller than the nuclear timescale. Furthermore, a binary is expected to become circularized during the RLOF. Alternatively, we also consider a uniform eccentricity distribution in the range $[0,1]$.

(5) We simply assume a constant SFR over the past 14 Gyr or, alternatively, as a delta function, i.e., a single instantaneous starburst. In the case of the constant SFR, we assume that one binary with a primary more massive than $0.8 M_{\odot}$ is formed annually (e.g., Iben \& Tutukov 1984; Han et al. 1995). For the case of the single starburst, we assume a burst producing $10^{11} M_{\odot}$ in stars, which is a typical mass of a galaxy. A galaxy may actually have a complicated star formation history. We only choose these two extremes for simplicity. A constant SFR is similar to the situation of spiral galaxies (Yungelson \& Livio 1998; Han \& Podsiadlowski 2004), whereas a delta function to that of elliptical galaxies or globular clusters.

\section{Results of binary population synthesis}

\subsection{Rates and delay times of $\mathrm{SNe}$ la}

The BPS studies are highly dependent on the chosen initial conditions in these Monte Carlo simulations. To systematically investigate Galactic SN Ia rates for the double-detonation model, six sets of simulations (see Table 1 ) with metallicity $Z=0.02$ are performed, where set 2 is our standard model with the best choice of model parameters (e.g., Wang et al. 2010b). The initial model parameters in other sets is varied to examine their influences on the final results. According to the six sets of Monte Carlo simulations, we find that the BPS is sensitive to uncertainties in some input parameters, in particular the mass-ratio distribution. If we adopt a mass-ratio distribution with un-correlated component masses (set 6), the SN Ia rate will decrease significantly, as most of the donors in the double-detonation model are not located inside the relevant contour of Fig. 2.

In Fig. 3, we show the evolution of Galactic SN Ia rates for the double-detonation model by adopting $Z=0.02$ and $S F R=$ $3.5 M_{\odot} \mathrm{yr}^{-1}$. The simulation for our standard model (set 2) gives a Galactic SN Ia rate of $\sim 1.5 \times 10^{-3} \mathrm{yr}^{-1}$, which is roughly one third of the inferred Galactic SN Ia rate $\left(3-4 \times 10^{-3} \mathrm{yr}^{-1}\right.$; Cappellaro \& Turatto 1997). This is in good agreement with the measured rates of SNe Iax $\left(31_{-13}^{+17} \mathrm{SNe}\right.$ Iax for every $100 \mathrm{SNe}$ Ia in a given volume; Foley et al. 2013). The birthrate from $\alpha_{\mathrm{ce}} \lambda=$ 0.5 (set 1) is lower than that of $\alpha_{\mathrm{ce}} \lambda=1.5$ (set 2), since the post-CE binaries are more likely to be located in the SN Ia production region for $\alpha_{\mathrm{ce}} \lambda=1.5$. As expected, these rates are consistent with the double-detonation model only producing part of

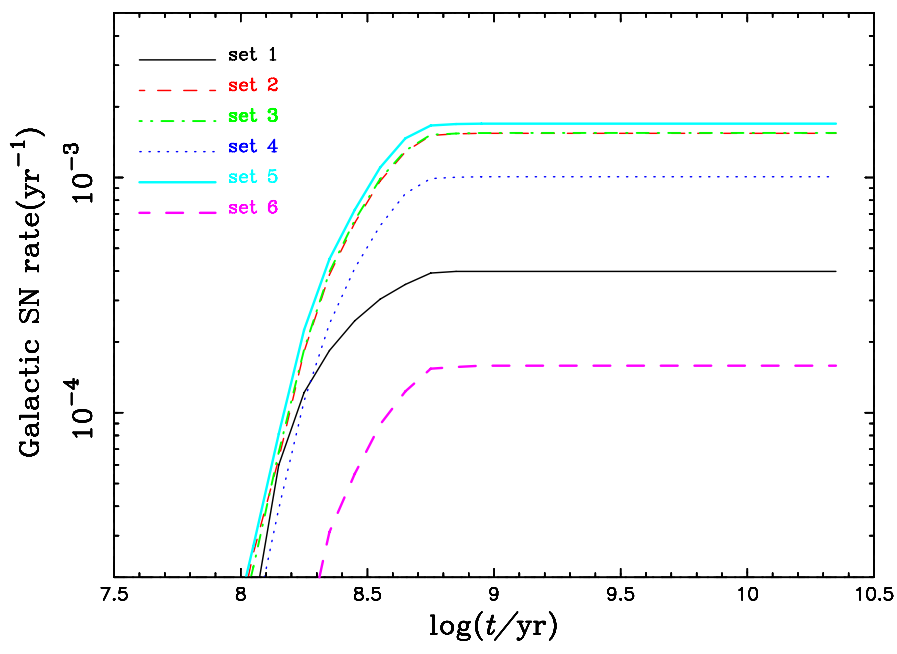

Fig. 3. Evolution of Galactic SN Ia rates for a constant Pop I SFR $\left(Z=0.02\right.$, SFR $\left.=3.5 M_{\odot} \mathrm{yr}^{-1}\right)$. The key to the line-styles representing different sets is given in the upper-left corner. The result of set 3 almost coincides with that of set 2 .

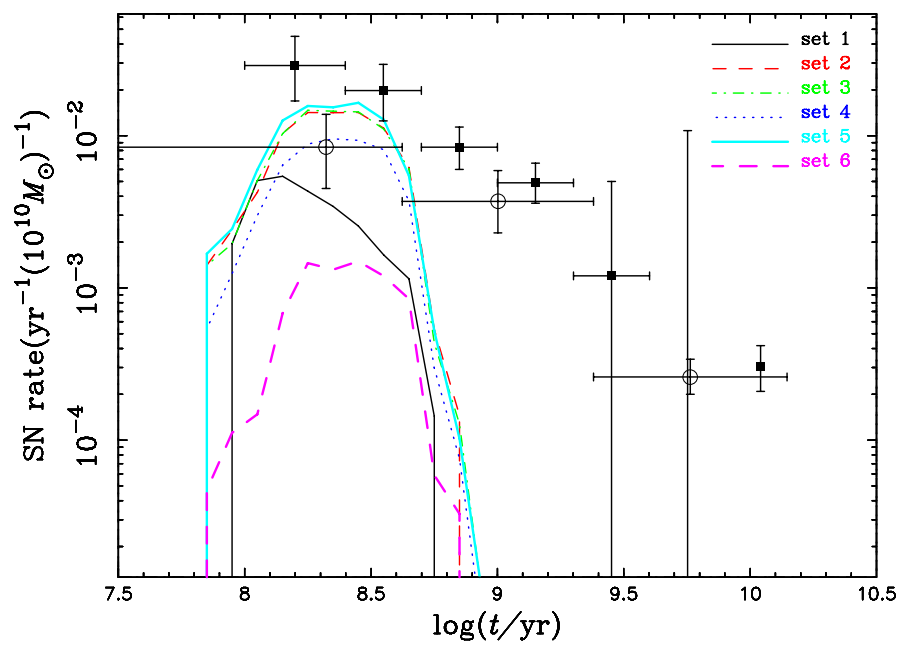

Fig. 4. Delay time distributions of $\mathrm{SNe}$ Ia for the double-detonation model. The filled squares and open circles are taken from Totani et al. (2008) and Maoz et al. (2011), respectively. The result of set 3 almost coincides with that of set 2 .

the overall SN Ia rate (for a recent review of other potential SN Ia formation channels see Wang \& Han 2012).

The delay times of SNe Ia are defined as the time interval between the star formation and SN explosion. The various progenitor models can be examined by comparing the delay time distributions with that of observations. Figure 4 displays the delay time distributions of SNe Ia for the double-detonation model. In the figure, we see that SN Ia explosions occur between $\approx 70 \mathrm{Myr}$ and $\approx 800 \mathrm{Myr}$ after the starburst, consistent with the current SNe Iax sample, most of which have originated in late-type, i.e., star-forming, galaxies. In Fig. 5, we show the delay time distributions of SNe Ia from different progenitor models. From this figure, we can see that the double-detonation sub-Chandrasekhar mass model is only a subclass for producing SNe Ia.

\subsection{Initial parameters of WD + He star systems}

To aid searches for candidate SN Ia progenitors, we show some properties of initial WD + He star systems which are predicted to produce SNe Ia based on these BPS calculations. Figure 6 presents the distribution of the initial orbital periods of the 


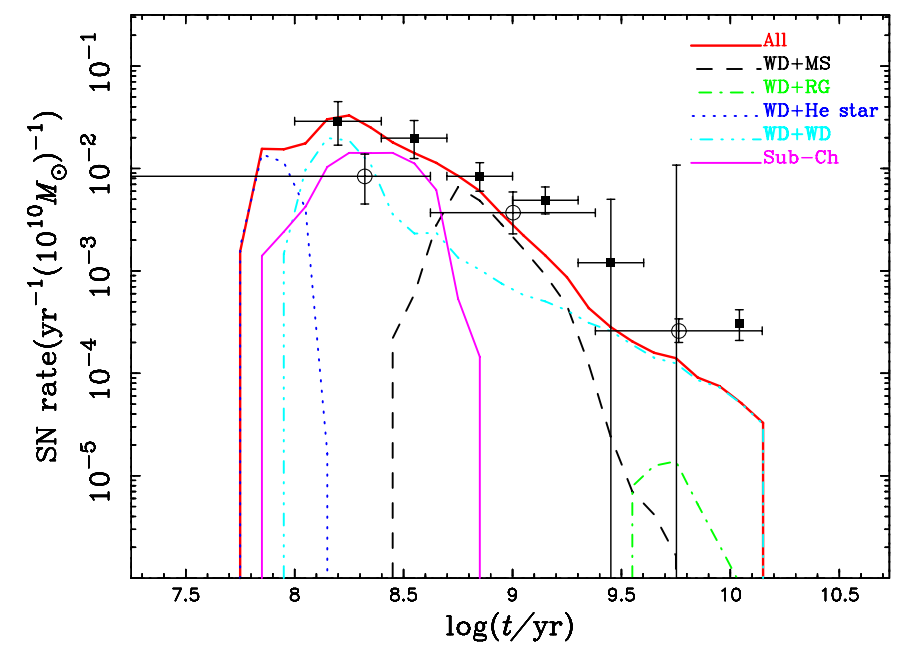

Fig. 5. Delay time distributions of SNe Ia for different progenitor models. The results for the WD + MS, WD + RG, WD + He star, and WD + WD models are from Wang et al. (2010b). The double-detonation subChandrasekhar mass model presents the result of set 2 .

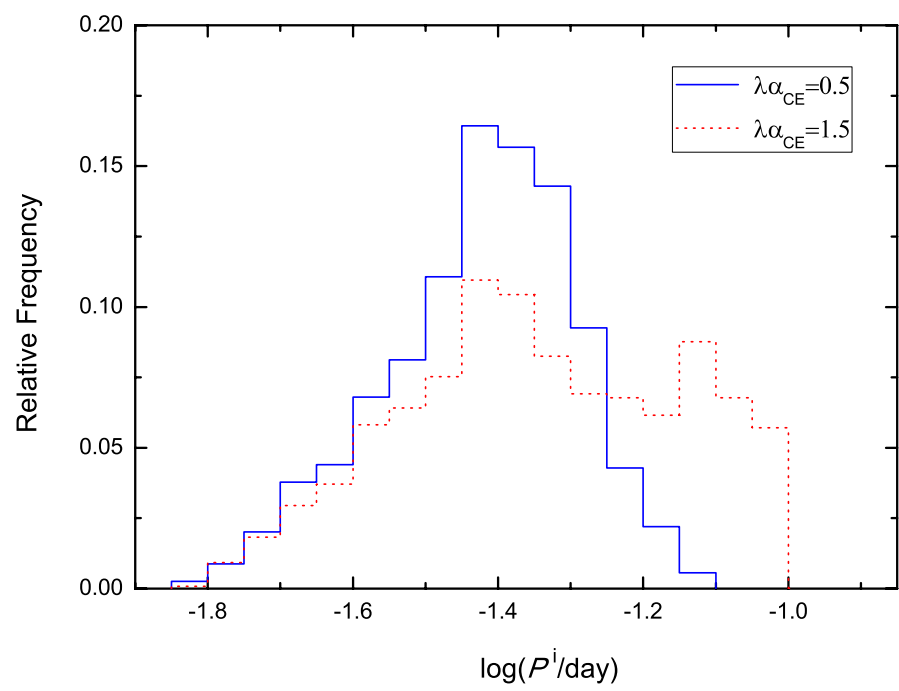

Fig. 6. Distribution of the initial orbital periods of the WD + He star systems that can ultimately produce SNe Ia. The solid and the dotted histograms represent the cases with $\alpha_{\mathrm{ce}} \lambda=0.5$ (set 1) and $\alpha_{\mathrm{ce}} \lambda=1.5$ (set 2), respectively.

$\mathrm{WD}+\mathrm{He}$ star systems that ultimately produce SNe Ia with different $\alpha_{\mathrm{ce}} \lambda$. These distributions are given at the current epoch, assuming a metallicity $z=0.02$ and an ongoing constant SFR. The figure illustrates that a higher value of $\alpha_{\mathrm{ce}} \lambda$ leads to wider $\mathrm{WD}+\mathrm{He}$ star binaries, as could be expected since a high value of $\alpha_{\text {ce }} \lambda$ allows the CE to be ejected with a smaller decrease in the orbit of the binary. Figure 7 shows the distribution of the masses of the WDs at formation, revealing that a low value of $\alpha_{\mathrm{ce}} \lambda$ tends to lead to higher initial WD masses. This trend can be understood by considering the He star channel (see Sect. 4), which allows stable RLOF to produce more massive WDs (when compared to dynamical mass transfer and a CE phase). Our BPS simulations find that a low value of $\alpha_{\mathrm{ce}} \lambda$ will increase the fraction of SNe Ia formed via the He star channel, and will therefore tend to produce more massive WDs. In Fig. 8, we display the distribution of the masses of the He donor stars as they are formed. A low value of $\alpha_{\mathrm{ce}} \lambda$ in this figure tends to produce larger He star masses. This is also related to the stable RLOF chanel, which initially

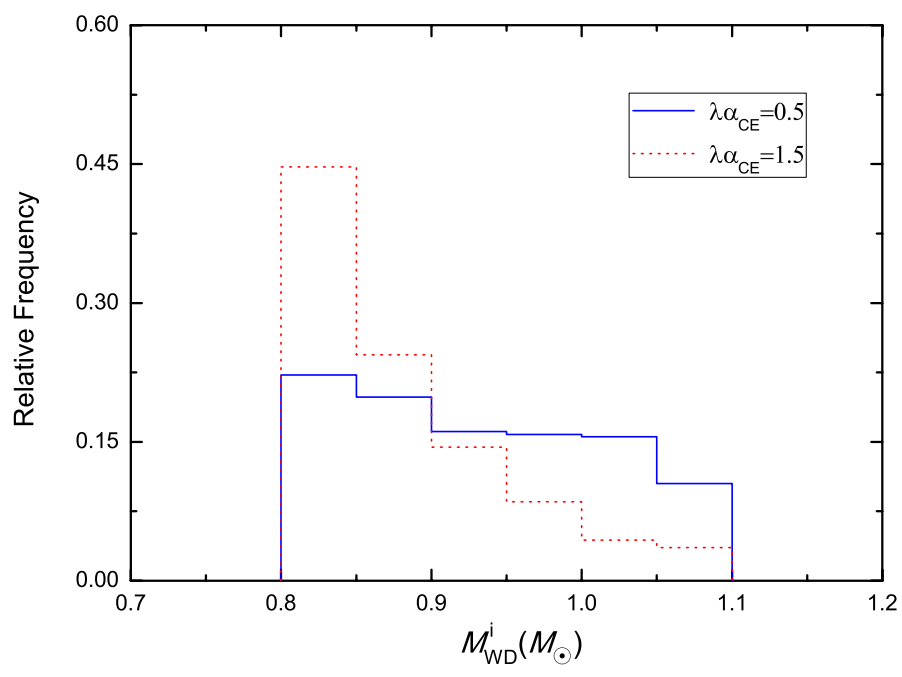

Fig. 7. Similar to Fig. 6, but for the distribution of the initial WD masses in the WD + He star systems.

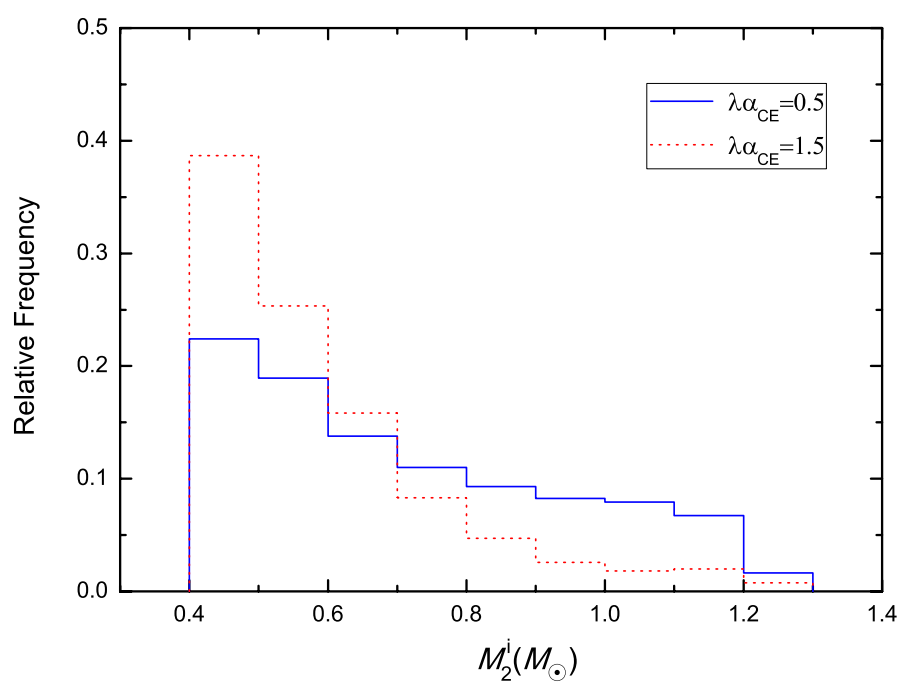

Fig. 8. Similar to Fig. 6, but for the distribution of the initial masses of secondaries in the WD + He star systems.

involves more massive companion stars and so typically leads to larger final He-core masses (and therefore He star masses).

According to our binary evolution calculations, most of the binaries have a SN ejecta-mass $\sim\left(M_{\mathrm{WD}}^{\mathrm{i}}+0.1 M_{\odot}\right.$ helium layer mass), except the binaries with a He donor mass $>1.1 M_{\odot}$. Because of orbit decay induced by GWR and large mass-ratio, the binaries with a He donor mass $>1.1 M_{\odot}$ experience a high mass-transfer rate, leading to the increase in the WD mass. However, binaries with a He donor mass above $>1.1 M_{\odot}$ only account for a small proportion (see Fig. 8), i.e., they have a small contribution for the distribution of the SN ejecta-mass.

\subsection{Luminosity distribution}

To quantify the relationship between the WD explosion mass $\left(M_{\mathrm{WD}}\right)$ and the SN Ia peak bolometric magnitude $\left(M_{\mathrm{bol}}\right)$, Ruiter et al. (2013) recently carried out a series of simulations for a range of WD masses based on 1D sub-Chandrasekhar mass pure detonation models, following the earlier similar calculations by Sim et al. (2010). We note that the Ruiter et al.' numbers are an upper limit on the luminosity, since deflagration tends to produce less luminosity than detonation (e.g., Khokhlov 1991). We also note that these simulations assumed a central detonation, with no 


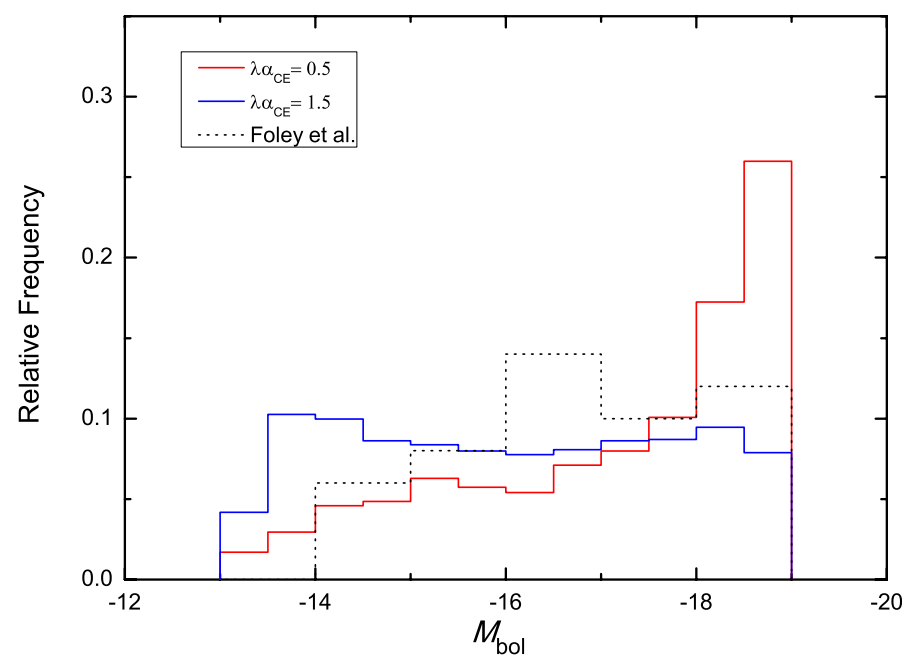

Fig. 9. Distribution of peak bolometric magnitudes from the population model (for two different $\alpha_{\mathrm{ce}} \lambda$ values). The data are observed SN Iax $V$-band magnitudes from Foley et al. (2013), several of which are lower limits.

specified triggering mechanism; we assume that their broad results are applicable to double-detonation events. The SN Ia peak brightness in their simulations is directly related to the WD explosion mass. We apply the $M_{\mathrm{WD}}-M_{\mathrm{bol}}$ relationship found by Ruiter et al. (2013) to derive the SN Ia peak brightness.

The resulting luminosity distribution is plotted in Fig. 9. There we also show observed peak V-band magnitudes from Foley et al. (2013), although several of these are lower limits and the sample is still probably too small to draw strong conclusions from the shape of the distribution. Nonetheless, the predicted peak magnitude range reproduces the full observed diversity of SNe Iax (-14.2 $\gtrsim M_{\mathrm{V}} \gtrsim-18.9 \mathrm{mag}$; Foley et al. 2013). The predicted range $\left(-13 \gtrsim M_{\text {bol }} \gtrsim-19 \mathrm{mag}\right.$ ) does add a tail stretching to fainter magnitudes, which might indicate a deficiency in the models or an observational bias against discovering the fainter $\mathrm{SNe}$ Iax. We note that these specific detonation models cannot be accurate models of SNe Iax, and if similar detonations can somehow lead to low explosion velocities then the physical modification which produces these low velocities would need to leave the luminosities unchanged.

\section{Discussion and conclusions}

The regions for producing SNe Ia (Fig. 2) depend on many uncertain input parameters, in particular the properties of the helium layer which is poorly known. In this article, we assume that a double-detonation is triggered when a helium layer with mass $0.1 M_{\odot}$ accumulates on the surface of the WD. However, it is not really known what the trigger point should be and so, to some extent, any choice is arbitrary. To check whether this choice affects the contours for producing $\mathrm{SNe} \mathrm{Ia}$, we also show the results of a $0.05,0.1$ and $0.2 M_{\odot}$ helium layer for a specific initial WD mass (see Fig. 10). From this figure, we see that the helium layer value has a significant influence on the contours for producing $\mathrm{SNe} \mathrm{Ia}$, and a lower value of helium layer mass has a bigger region. This is because the fact that a lower value of helium layer mass needs less mass from the helium donor star.

The most relevant known binary system to our current work is perhaps CD-3011223, which has been identified as a COWD + sdB star system with a $\sim 1.2 \mathrm{~h}$ orbital period (Vennes et al. 2012; Geier et al. 2013). Geier et al. (2013) constrained both the mass of the $\mathrm{sdB} \sim 0.51 M_{\odot}$ and the mass of the WD companion $\sim 0.76 M_{\odot}$. Owing to the short orbital period, angular

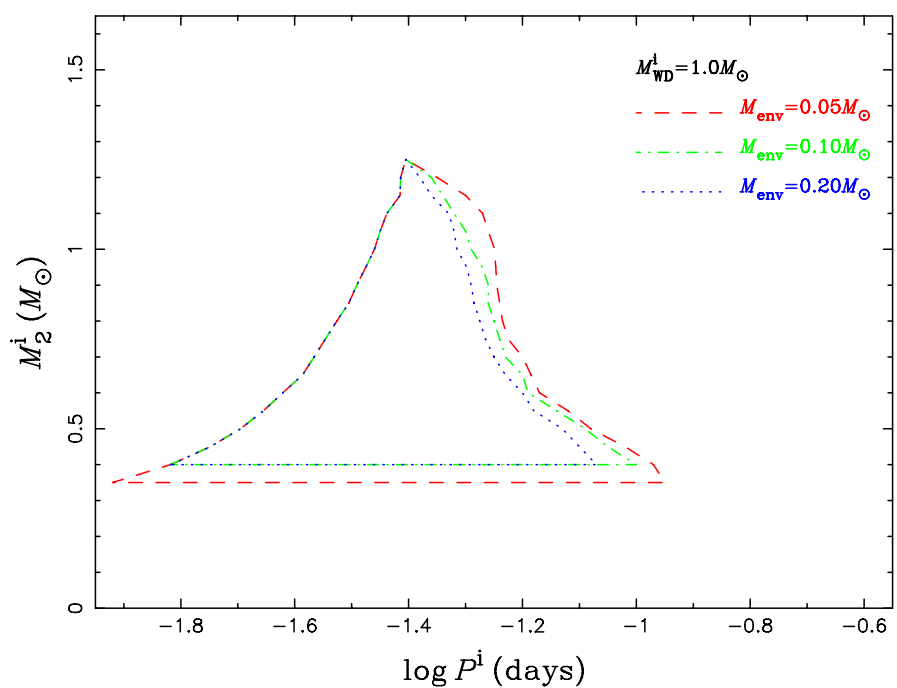

Fig. 10. Regions in the initial orbital period-secondary mass plane $\left(\log P^{\mathrm{i}}, M_{2}^{\mathrm{i}}\right)$ for WD $+\mathrm{He}$ star binaries that produce SNe Ia with initial WD mass of $1.0 M_{\odot}$, but for different helium layer masses.

momentum loss from GWR is large. After about 36 million years, the sdB star will begin to fill its Roche lobe whilst it is still in the He-core burning stage. The binary system might produce an SN Ia via the double-detonation model during its future evolution (e.g., Geier et al. 2013). Other known CO WD + He-donor systems (e.g., KPD 1930+2752, V445 Pup, and HD 49798 with its WD companion) may produce $\mathrm{SNe}$ Ia via other progenitor models (see Wang \& Han 2012).

The donor star would survive the SN explosion and would potentially be identifiable soon after the WD is disrupted (e.g., Wang \& Han 2010b; Pan et al. 2010; Liu et al. 2012). Identification of these remnant objects would help to support this scenario. The surviving companions from the model might also explain hypervelocity helium-rich stars like US 708 (e.g., Hirsch et al. 2005) because of the short orbital periods at the moment of SN explosion (see also Justham et al. 2009; Wang \& Han 2009) ${ }^{4}$. Geier et al. (2013) suggested that the CO WD + sdB system CD-3011223 and the hypervelocity star US 708 might represent two different stages of an evolutionary sequence linked by an SN Ia explosion (the orbital velocity of the sdB at the moment of SN explosion will be about $600 \mathrm{~km} \mathrm{~s}^{-1}$ and thus close to the Galactic escape velocity). Studying these high-velocity heliumrich stars (and their WD descendants) might provide a way to test this model.

In this article, we have systematically studied a potential progenitor population for SNe Iax in which a non-degenerate helium donor star leads to a helium-ignited explosion of a subChandrasekhar CO WD. (1) This model can naturally produce SN explosions with helium lines and without hydrogen lines. (2) The event rate agrees with the inferred rates of SNe Iax. (3) The explosions in this model occur between $\approx 70 \mathrm{Myr}$ and $\approx 800 \mathrm{Myr}$ after the starburst, i.e., in relatively young stellar populations, consistent with the host galaxy morphologies of most of SNe Iax. (4) By adopting an existing relationship

4 An alternative explanation for the origin of US 708 is the merger model, in which US 708 was formed by the merger of two He-WDs in a close binary which was induced as they were ejected by the interaction with the super-massive black hole in the Galactic center (e.g., Hirsch et al. 2005); the age of US 708 makes this original scenario somewhat fine-tuned. Perets (2009) recently argued that US 708 might have been ejected as a binary from a triple disruption by the super-massive black hole, which later on evolved and merged to form an sdO star. 
between CO mass and SN luminosity for pure detonations, we find a SN luminosity range from -13 mag to -19 mag, which compares well to the current diversity of SNe Iax $(-14.2 \gtrsim$ $M_{\mathrm{V}} \gtrsim-18.9 \mathrm{mag}$ ). The overall population properties resulting from this model therefore seem promisingly consistent with the known collection of most of SNe Iax. However, the current models of double detonation have difficulty in producing the observed low-velocity ejecta characteristic of SNe Iax, and they cannot produce low ejecta-mass for SN 2008ha as inferred in the introduction.

Current models typically predict that a double-detonation explosion will follow the ignition of the He-layer, and we have generally assumed that to be the case. However, we reiterate that there seems to be sufficient uncertainty in these explosion models to allow other outcomes. Our results are more suggestive than definitive, but confirmation that helium double-detonation explosions can produce a form of SNe Ia would finally answer a fascinating astrophysical question, and help to constrain our understanding of explosion physics.

If these progenitors do produce SNe Iax, it would not exclude other double-detonation events from producing some normal $\mathrm{SNe}$ Ia. However, this would suggest a new problem: why would systems with non-degenerate helium donors make SNe Iax but not - or only rarely - those with He WD donors? We can only speculate about potential answers. Degenerate donors tend to produce higher mass-transfer rates than our non-degenerate donors (e.g., Han \& Webbink 1999), and higher mass-transfer rates require less massive helium layers for ignition (e.g., Shen $\&$ Bildsten 2009). One possibility is that current theoretical work underestimates the mass of helium layer which is required to lead to a double detonation, in which case the less massive helium-shell detonations do not lead to a form of SNe Ia. Alternatively, if double detonations are triggered in systems with lower-mass helium layers, then perhaps only the more massive helium shells sufficiently alter the explosion properties for them to be identified as peculiar, 2002cx-like, events. Or perhaps helium layers which are too thick do not produce double detonations after all; SNe Iax do require lower ejecta velocities than the double-detonation explosion models currently predict, and perhaps this can be achieved if massive enough helium layers can ignite $\mathrm{CO}$ deflagrations. We intend to study the potential diversity of this population in future by adopting more sophisticated assumptions about the properties of the helium layer at ignition. The 2002cx-like subclass of SNe Ia certainly deserves further detailed study.

Acknowledgements. We acknowledge the anonymous referee for the valuable comments that helped us to improve the paper. We also thank useful comments and suggestions from Hagai Perets. This work is partly supported by the 973 programme of China (No. 2014CB845703), the NSFC (Nos. 11322327, 11103072, 11033008, 11250110055 and 11350110324), the CAS (No. KJCX2-YW-T24), and the Talent Project of Young Researchers of Yunnan province.

\section{References}

Benetti, S., Cappellaro, E., Mazzali, P. A., et al. 2005, ApJ, 623, 1011 Bildsten, L., Shen, K. J., Weinberg, N. N., \& Nelemans, G. 2007, ApJ, 662, L95 Branch, D., Livio, M., Yungelson, L. R., Boffi, F. R., \& Baron, E. 1995, PASP, 107,1019

Cappellaro, E., \& Turatto, M. 1997, in Thermonuclear Supernovae, eds. P. Ruiz-Lapuente, R. Canal, \& J. Isern (Dordrecht: Kluwer), 77

Eggleton, P. P. 1971, MNRAS, 151, 351

Eggleton, P. P. 1972, MNRAS, 156, 361

Eggleton, P. P. 1973, MNRAS, 163, 279

Eggleton, P. P., Tout, C. A., \& Fitechett, M. J. 1989, ApJ, 347, 998

Fink, M., Hillebrandt, W., \& Röpke, F. K. 2007, A\&A, 476, 1133

Fink, M., Röpke, F. K., Hillebrandt, W., et al. 2010, A\&A, 514, A53

Foley, R. J., Chornock, R., Filippenko, A. V., et al. 2009, AJ, 138, 376
Foley, R. J., Brown, P. J., Rest, A., et al. 2010, ApJ, 708, L61 Foley, R. J., Challis, P. J., Chornock, R., et al. 2013, ApJ, 767, 57 Geier, S., Marsh, T. R., Wang, B., et al. 2013, A\&A, 554, A54 Goldberg, D., \& Mazeh, T. 1994, A\&A, 282, 801

Hachinger, S., Mazzali, P. A., Taubenberger, S., et al. 2012, MNRAS, 422, 70 Han, Z., \& Podsiadlowski, Ph. 2004, MNRAS, 350, 1301

Han, Z., \& Webbink, R. F. 1999, A\&A, 349, L17

Han, Z., Podsiadlowski, Ph., \& Eggleton, P. P. 1994, MNRAS, 270, 121

Han, Z., Podsiadlowski, Ph., \& Eggleton, P. P. 1995, MNRAS, 272, 800

Han, Z., Tout, C. A., \& Eggleton, P. P. 2000, MNRAS, 319, 215

Hillebrandt, W., \& Niemeyer, J. C. 2000, ARA\&A, 38, 191

Hirsch, H. A., Heber, U., O'Toole, S. J., \& Bresolin, F. 2005, A\&A, 444, L61

Höflich, P., \& Khokhlov, A. 1996, ApJ, 457, 500

Hurley, J. R., Pols, O. R., \& Tout, C. A. 2000, MNRAS, 315, 543

Hurley, J. R., Tout, C. A., \& Pols, O. R. 2002, MNRAS, 329, 897

Iben, I., \& Tutukov, A. V. 1984, ApJS, 54, 335

Ivanova, N., \& Taam, R. E. 2004, ApJ, 601, 1058

Jordan IV, G. C., Perets, H. B., Fisher, R. T., \& van Rossum, D. R. 2012, ApJ, $761, \mathrm{~L} 23$

Justham, S., Wolf, C., Podsiadlowski, P., \& Han, Z. 2009, A\&A, 493, 1081

Kasliwal, M. M., Kulkarni, S. R., Gal-Yam, A., et al. 2010, ApJ, 723, L98

Kasliwal, M. M., Kulkarni, S. R., Gal-Yam, A., et al. 2012, ApJ, 755, 161

Khokhlov, A. M. 1991, A\&A, 245, 114

Kromer, M., Sim, S. A., Fink, M., Röpke, F. K., Seitenzahl, I. R., \& Hillebrandt, W. 2010, ApJ, 719, 1067

Kromer, M., Fink, M., Stanishev, V., et al. 2013, MNRAS, 429, 2287

Kroupa, P., Tout, C. A., \& Gilmore, G. 1993, MNRAS, 262, 545

Landau, L. D., \& Lifshitz, E. M. 1971, Classical theory of fields (Oxford: Pergamon Press)

Li, W., Filippenko, A. V., Chornock, R., et al. 2003, PASP, 115, 453

Liu, Z., Pakmor, R., Röpke, F. K., et al. 2012, A\&A, 548, A2

Livne, E. 1990, ApJ, 354, L53

Lyman, J. D., James, P. A., Perets, H. B., et al., 2013, MNRAS, 434, 527

Maoz, D., Mannucci, F., Li, W., et al. 2011, MNRAS, 412, 1508

Mazeh, T., Goldberg, D., Duquennoy, A., \& Mayor, M. 1992, ApJ, 401, 265

Miller, G. E., \& Scalo, J. M. 1979, ApJS, 41, 513 (MS79)

Moll, R., \& Woosley, S. E. 2013, ApJ, 774, 137

Nomoto, K. 1982, ApJ, 257, 780

Pan, K.-C., Ricker, P. M., \& Taam, R. E. 2010, ApJ, 715, 78

Perets, H. B. 2009, ApJ, 698, 1330

Perets, H. B., Gal-Yam, A., Mazzali, P. A., et al. 2010, Nature, 465, 322

Perets, H. B., Badenes, C., Arcavi, I., et al. 2011, ApJ, 730, 89

Pols, O. R., Schröder, K. P., Hurly, J. R., Tout, C. A., \& Eggleton, P. P. 1998, MNRAS, 298, 525

Poznanski, D., Chornock, R., Nugent, P. E., et al. 2010, Science, 327, 58 Raghavan, D., McAlister, H. A., Henry, T. J., et al. 2010, ApJS, 190, 1 Ruiter, A. J., Belczynski, K., Sim, S. A., et al. 2011, MNRAS, 417, 408

Ruiter, A. J., Sim, S. A., Pakmor, R., et al. 2013, MNRAS, 429, 1425

Sana, H., de Mink, S. E., de Koter, A., et al. 2012, Science, 337, 444

Scalo, J. M. 1986, Fund. Cosm. Phys., 11, 1 (S86)

Shatsky, N., \& Tokovinin, A. 2002, A\&A, 382, 92

Shen, K. J., \& Bildsten, L. 2009, ApJ, 699, 1365

Shen, K. J., \& Bildsten, L. 2013, ApJ, submitted [arXiv: 1305. 6925]

Sim, S. A., Röpke, F. K., Hillebrandt, W., et al. 2010, ApJ, 714, L52

Sim, S. A., Fink, M., Kromer, M., et al. 2012, MNRAS, 420, 3003

Sullivan, M., Kasliwal, M. M., Nugent, P. E., et al. 2011, ApJ, 732, 118

Totani, T., Morokuma, T., Oda, T., Doi, M., \& Yasuda, N. 2008, PASJ, 60, 1327

Valenti, S., Pastorello, A., Cappellaro, E., et al. 2009, Nature, 459, 674

Vennes, S., Kawka, A., O’Toole, S. J., Németh, P., \& Burton, D. 2012, ApJ, 759, L25

Waldman, R., Sauer, D., Livne, E., et al. 2011, ApJ, 738, 21

Wang, B., \& Han, Z. 2009, A\&A, 508, L27

Wang, B., \& Han, Z. 2010a, A\&A, 515, A88

Wang, B., \& Han, Z. 2010b, MNRAS, 404, L84

Wang, B., \& Han, Z. 2012, New Astron. Rev., 56, 122

Wang, B., Meng, X., Chen, X., \& Han, Z. 2009a, MNRAS, 395, 847

Wang, B., Chen, X., Meng, X., \& Han, Z. 2009b, ApJ, 701, 1540

Wang, B., Li, X.-D., \& Han, Z. 2010a, MNRAS, 401, 2729

Wang, B., Liu, Z., Han, Y., et al. 2010b, Sci. China Ser. G, 53, 586

Webbink, R. F. 1984, ApJ, 277, 355

Woosley, S. E., \& Kasen, D. 2011, ApJ, 734, 38

Woosley, S. E., \& Weaver, T. A. 1994, ApJ, 423, 371

Woosley, S. E., Taam, R. E., \& Weaver, T. A. 1986, ApJ, 301, 601

Yoon, S. C., \& Langer, N. 2004, A\&A, 419, 645

Yuan, F., Kobayashi, C., Schmidt, B. P., et al. 2013, MNRAS, 432, 1680

Yungelson, L., \& Livio, M. 1998, ApJ, 497, 168

Zoccali, M., Cassisi, S., Frogel, J. A., et al. 2000, ApJ, 530, 418 